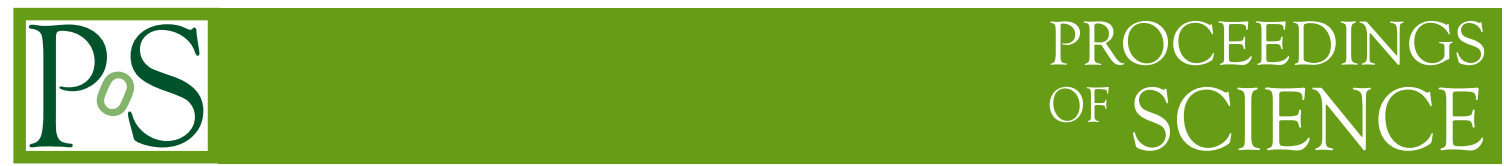

\title{
X-ray spectroscopy of GRO J1655-40
}

\section{Gloria Sala ${ }^{* \dagger}$}

Max-Planck-Institut für extraterrestrische Physik, Garching, Germany

E-mail: gsala@mpe.mpg.de

\section{Jochen Greiner}

Max-Planck-Institut für extraterrestrische Physik, Garching, Germany

E-mail: jcg@mpe.mpg.de

\section{Jacco Vink}

Astronomical Institute, University Utrecht, The Netherlands

E-mail: J.Vink@astro.uu.nl

\section{Frank Haberl}

Max-Planck-Institut für extraterrestrische Physik, Garching, Germany

E-mail: fwh@mpe.mpg.de

\begin{abstract}
After seven years in quiescence, GRO J1655-40 started a new outburst in February 2005, rising to a high/soft state in March 2005. We report here the results of four XMM-Newton observations performed on 27 February 2005 in the low/hard state, and on three consecutive days in March 2005 during the rise of the source to its high/soft state. The X-ray spectrum of GRO J1655-40 was dominated in the high/soft state by the thermal emission from the accretion disk, with a maximum temperature of $1.3 \mathrm{keV}$. We detected two absorption lines in the EPIC-pn spectra at 6.7-6.8 and 7.8-8.0 keV, which could be identified as the $\mathrm{K} \alpha$ and $\mathrm{K} \beta$ lines of either blended Fe XXV and Fe XXVI or blueshifted Fe XXV. We found no orbital dependence on the X-ray properties, which provides an upper limit for the inclination of the system of $73^{\circ}$.
\end{abstract}

VI Microquasar Workshop: Microquasars and Beyond September 18-22, 2006

Como, Italy

\footnotetext{
*Speaker.

${ }^{\dagger}$ Postdoctoral fellow at MPE supported by the Spanish Ministry of Education and Science.
} 


\section{Introduction}

The microquasar GROJ1655-40 [1] was the second superluminal source discovered in our Galaxy after GRS 1915+105 [2]. The two sources may also be peculiar in the sense that both systems show evidence of containing a maximally spinning black hole [3]. Radio images of GRO J1655-40 showed twin jets with apparent superluminal motion moving in opposite directions at $0.92 \mathrm{c}$ [ [ $\boxminus$, and the distance was determined to be $3.2 \pm 0.2 \mathrm{kpc}$ [5]. Using the dust scattering halo observed by ROSAT, a distance of $3 \mathrm{kpc}$ was determined [6], compatible with the previous determinations. However, a recent new determination of the distance locates GRO J1655-40 at less than $1.7 \mathrm{kpc}$ [7].

Optical observations in 1996 provided an inclination angle of $65.5 \pm 0.08$, a radial velocity semiamplitude of $228.2 \pm 2.2 \mathrm{~km} \mathrm{~s}^{-1}$, and a dynamical mass of the primary component of $7.02 \pm 0.22 \mathrm{M}_{\odot}[8]$, indicating that it is a black hole.

After 7 years of inactivity, GRO J1655-40 left quiescence again on 17 February 2005 [?]. The X-ray evolution was followed with RXTE/ASM [10] and Swift [11]. In February GRO J165540 entered a low/hard state [12], and at the beginning of March it experienced a rise in flux and entered into the high/soft state, reaching $\sim 2$ Crab on 19 March 2005 (Fig. 11 left panel). Here we present XMM-Newton observations performed in February-March 2005, aimed at obtaining detailed spectroscopy during the rise to the high/soft state.

\section{Observations and spectral analysis}

We observed GRO J1655-40 with XMM-Newton some days before the rise to the soft/high state on 27 February 2005 (40 ks; obs. id. 0112921-301) and again on 14, 15, and 16 March 2005, close to the maximum of the first outburst (15 ks each, obs. id. 0112921-401/-502/-601). The XMM-Newton data were reduced using SAS 6.1, and XSPEC 11.3 was used for spectral analysis. The high luminosity of GRO J1655-40 at the time of our XMM-Newton observations (almost twice as bright as the Crab, the source used for the burst mode calibration) made evident a ratedependence of the charge transfer efficiency (CTE) that was not included in the calibration. We determined this dependence and corrected our spectra accordingly. Simultaneous RXTE observations of GRO J1655-40 are publicly available. We have included the standard spectral products from the PCA and HEXTE instruments in our analysis, to constrain the model components at hard $\mathrm{X}$-rays. More details on the observations and data reduction can be found in [13].

During the first observation on 27 February 2005, before the start of the first outburst, the source was in a low/hard state, with the XMM-Newton plus RXTE instruments showing a spectrum dominated by an absorbed power law with photon index $\Gamma=1.48 \pm 0.01$ (uncorrelated $90 \%$ error for 1 degree of freedom). The fit with a power law leaves excess residuals at low energies both in the EPIC-pn and the RGS spectra, but the fit improves when the spectrum is modified by the scattering of dust along the line-of-sight. In ROSAT HRI observations in 1994, a halo was found around GRO J1655-40 due to the scattering of the X-rays by interstellar dust [6]. We thus modify our spectral model with the dust model available in XSPEC, which assumes that the scattered flux appears as a uniform disk on the sky (as for a source that is constant in time) whose size has a 1/E dependence and whose total flux has a $1 / \mathrm{E}^{2}$ dependence. 

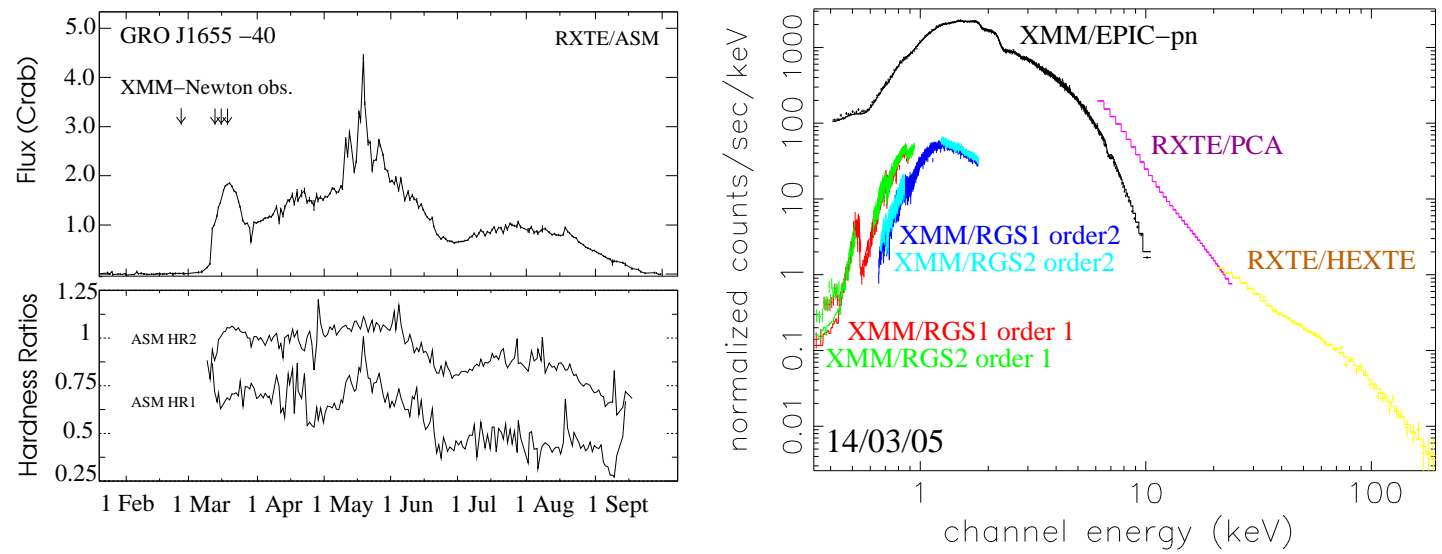

Figure 1: Left panel: RXTE/ASM light-curve of GRO J1655-40 during 2005. The dates of our XMMNewton observations are indicated by arrows. Right panel: XMM-Newton and RXTE observed spectra on 14 March 2005.

The RXTE/PCA spectrum also shows residuals between 6-7 keV, which are well-fitted with an iron fluorescence line with energy fixed at $6.4 \mathrm{keV}$, for which we derive an equivalent width of $135 \pm 20 \mathrm{eV}$. The line is not seen in the EPIC-pn data, and by fixing the energy at $6.4 \mathrm{keV}$, we obtain an upper limit of $140 \mathrm{eV}$ for the equivalent width. The presence of an iron fluorescence line in the low/hard state is confirmed by Swift observations one week after our XMM-Newton observation [11].

The three March observations have very similar spectra, with the XMM-Newton continuum spectra dominated by an absorbed multicolor disk (MCD), where $\mathrm{kT}_{\text {in }}$ increases with time from 1.25 to $1.35 \mathrm{keV}$, and the higher energy spectra from RXTE represented by a soft power law, with $\Gamma=2.1-2.3$ (Fig. 1, right panel; Table 1). The simultaneous fit of the EPIC-pn, RGS, PCA, and HEXTE data with the MCD-plus-power-law model leaves large residuals again below $0.6 \mathrm{keV}$ in both the EPIC-pn and the RGS spectra. The soft excess in the XMM-Newton instruments, as in the case of the February observation, is most probably related to the dust scattering. Fixing the dust halo size to 10 times the beam size, from the fit with the EPIC-pn data, we obtain in all cases a scattering fraction at $1 \mathrm{keV}$ of $17-20 \%$, similar to the $21 \%$ relative intensity of the halo found with ROSAT [6].

\section{Results and discussion}

The spectrum of GRO J1655-40 during our March 2005 observations is dominated by the thermal emission of the accretion disk plus a power law component at high energies. The maximum disk temperature, increasing from 1.25 to $1.35 \mathrm{keV}$, is consistent with the values commonly observed in black-hole binaries in the high/soft state and in GRO J1655-40 with Swift and INTEGRAL during the same period [11, 14]. The inner radius, $\mathrm{R}_{\mathrm{in}} \sim 13-14[\mathrm{D} / 3.2 \mathrm{kpc}] \mathrm{km}$, is only a little larger than the gravitational radius of the black hole, $\mathrm{R}_{\mathrm{g}}=\mathrm{GM} / \mathrm{c}^{2}=10 \mathrm{~km}$, consistent with a rapidly spinning black hole [邚.

A new determination for the distance to GRO J1655-40 has been recently obtained [7] and indicates an upper limit of $1.7 \mathrm{kpc}$. In this case, the luminosity in the range $0.4-10 \mathrm{keV}$ would be 
Table 1: Continuum spectral model for XMM-Newton and RXTE observations

\begin{tabular}{ccccc}
\hline \hline Parameters & 27 Feb 2005 & 14 March 2005 & 15 March 2005 & 16 March 2005 \\
\hline $\mathrm{N}_{\mathrm{H}}\left(\times 10^{21}\right) \mathrm{cm}^{-2}$ & $5.2 \pm 0.2$ & $5.46 \pm 0.02$ & $5.57 \pm 0.02$ & $5.45 \pm 0.03$ \\
Dust fraction $^{\mathrm{a}}$ & $0.15 \pm 0.03$ & $0.186 \pm 0.002$ & $0.169 \pm 0.003$ & $0.184 \pm 0.003$ \\
$\mathrm{kT}_{\text {in }}(\mathrm{keV})$ & - & $1.231 \pm 0.003$ & $1.268 \pm 0.003$ & $1.326 \pm 0.003$ \\
$\mathrm{R}_{\text {in }}^{\mathrm{b}}(\mathrm{km})$ & - & $14.25 \pm 0.05$ & $13.25 \pm 0.05$ & $13.55 \pm 0.05$ \\
$\Gamma$ & $1.48 \pm 0.01$ & $2.10 \pm 0.01$ & $2.27 \pm 0.01$ & $2.13 \pm 0.01$ \\
$\mathrm{~K}^{\mathrm{c}}\left(\mathrm{ph} \mathrm{keV}^{-1} \mathrm{~cm}^{-2} \mathrm{~s}^{-1}\right)$ & $0.0385 \pm 0.0007$ & $0.94 \pm 0.02$ & $0.76 \pm 0.07$ & $1.05 \pm 0.02$ \\
$\mathrm{~L}_{0.4-10 ~ \mathrm{deV}}^{\mathrm{d}}\left(\mathrm{erg} \mathrm{s}^{-1}\right)$ & $3.7 \times 10^{35}$ & $3.4 \times 10^{37}$ & $3.4 \times 10^{37}$ & $4.2 \times 10^{37}$ \\
\hline
\end{tabular}

${ }^{\text {a }}$ Dust scattering fraction at $1 \mathrm{keV}$. Halo size is fi xed to 10 times the detector beam-size at $1 \mathrm{keV}$.

${ }^{\mathrm{b}}$ For a distance of $3.2 \mathrm{kpc}$ and an inclination of $70^{\circ}$.

${ }^{\mathrm{c}}$ Normalization constant for the power law component; flux per keV @ $1 \mathrm{keV}$.

${ }^{\mathrm{d}}$ Luminosity in the range $0.4-10 \mathrm{keV}$, for a distance of $3.2 \mathrm{kpc}$.

$1.0 \times 10^{35}, 9.6 \times 10^{36}, 9.6 \times 10^{36}$, and $1.2 \times 10^{37} \mathrm{erg} \mathrm{s}^{-1}$ (for days $27 \mathrm{Feb}, 14,15$, and $16 \mathrm{March}$ respectively), and the disk inner radius would be only $7.6 \mathrm{~km}, 7.0 \mathrm{~km}$, and $7.2 \mathrm{~km}$ (for days 14,15 , and 16 March 2005). We note, however, that these values are lower than the gravitational radius of the black hole, $\mathrm{R}_{\mathrm{g}}=\mathrm{GM} / \mathrm{c}^{2} \sim 10 \mathrm{~km}$ (for $\mathrm{M}_{\mathrm{BH}}=7 \mathrm{M}_{\odot}$ as determined by [8]).

From the fit parameters of the MCD model, the luminosity of the disk can be calculated as $\mathrm{L}_{\mathrm{x}}=$ $4 \pi \mathrm{R}_{\text {in }}^{2} \sigma \mathrm{T}_{\text {in }}^{4}$ [15]. With our values, the accretion disk luminosity is $6-7 \times 10^{37}[\mathrm{D} / 3.2 \mathrm{kpc}]^{2} \mathrm{erg} \mathrm{s}^{-1}$. With the accretion disk luminosity, the accretion rate can be calculated as $2 \mathrm{~L}_{\mathrm{x}} \mathrm{R}_{\mathrm{in}} / \mathrm{GMg}^{2}$ [15], where $g=\left(1-R_{g} / R_{i n}\right)^{1 / 2}$ is a correction for the general relativity, $R_{g}$ the gravitational radius, and $\mathrm{M}$ the mass of the black hole. From the accretion-disk luminosity, the disk inner radius, and the black-hole mass of GRO J1655-40, we obtain an accretion rate during the high/soft state of $\sim 10^{-8} \mathrm{M}_{\odot} \mathrm{yr}^{-1}$.

The rise of the disk blackbody component during the high/soft state in March is reflected in the power law index of the corona, which increases from $1.48 \pm 0.01$ in February 27 to values higher than 2 in the March observations. This strong increase of the photon index was also observed by INTEGRAL [14]. With the rise in the thermal disk emission, the soft luminosity increases and the electrons in the corona are cooled more efficiently, resulting in a softer spectrum for the comptonized photons, i.e., a steeper power law.

\section{Interstellar absorption}

In the three March observations, the fit of the RGS spectra around the interstellar neutral oxygen edge at 22.6-23.2 A (534-549 eV) with the phabs absorption model is unacceptable unless the "Vern" cross-sections [16] available in XSPEC are used. Even in this case, the fit leaves clear residuals, indicating the presence of an additional absorption line at $23.5 \mathrm{~A}$ (Fig. 2. right panel), which corresponds to the $1 \mathrm{~s}-2 \mathrm{p}$ absorption line of neutral oxygen. Given the energy resolution of the RGS, the observed maximum width of the line is compatible with it being a narrow line originating in the interstellar medium. The RGS spectra also show clear absorption features at 

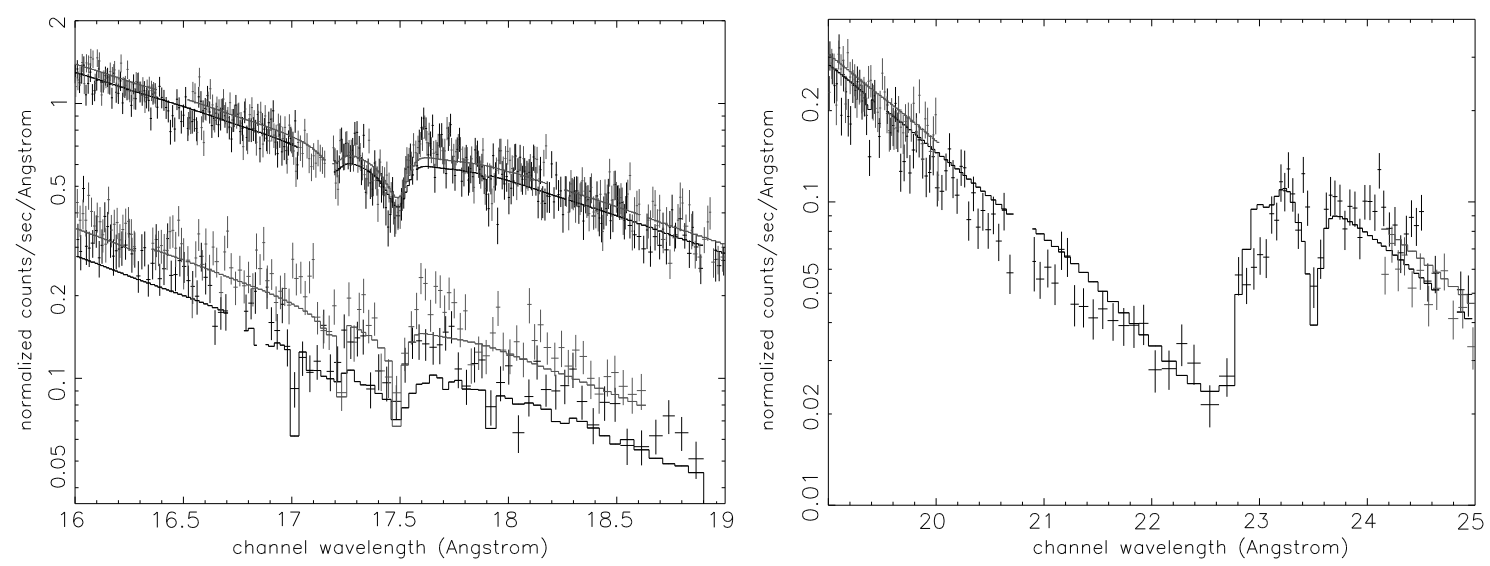

Figure 2: Left panel: First and second order (with lower count rate) spectra of GRO J1655-40 in the range 16-19 A, obtained with RGS1 (black) and RGS2 (grey) on 14 March 2005, showing the ISM Fe L edges fitted with the new TBabs model. Right panel: RGS1 (black) and RGS2 (grey, contributing only below $20 \mathrm{~A}$ and above $24 \mathrm{~A}$ ) around the oxygen edge in the first-order spectra of the 14 March 2005 observation, fitted with the new TBabs model.

17.2 A and 17.5 A (Fig. 2, left panel), which correspond to Fe $\mathrm{L}_{2,3}\left(2 \mathrm{p}_{1 / 2}, 2 \mathrm{p}_{3 / 2}\right)$ edges, also observed in the interstellar absorption towards some other sources [17, 18, 19]. As in these previous cases, simple edge models do not provide a good fit for these features.

Since both the line at 23.5 A and the edges at 17.2 A and 17.5 A are caused by interstellar gas, but are not reproduced well by the simple phabs model in XSPEC, the recent new version of the TBabs model [20] has been used. This has been shown to provide a good fit to the complex oxygen edge [21], including two edges and five absorption lines, and to the iron absorption features [22]. It also provides a good fit for our RGS features (Fig. 2), with a hydrogen column $\mathrm{N}_{\mathrm{H}}$ : $7.3( \pm 0.3), 7.0( \pm 0.3), 6.9( \pm 0.5) \times 10^{21} \mathrm{~cm}^{-2}$, and an overabundance of oxygen with respect to solar of 1.45( \pm 0.15$), 1.6( \pm 0.1), 1.45( \pm 0.15)$ (for days 14, 15, and 16 March 2005, respectively).

The interstellar OI K $\alpha$ line is clearly detected at 23.5 A in our three March 2005 RGS spectra. This line was also found in the RGS spectra of other sources and distinguished from the instrumental components around the interstellar oxygen edge, at 23.05 and $23.35 \mathrm{~A}$ [23]. The same line was detected in the high resolution spectra of seven X-ray binaries using the Chandra/HETGS [21], as part of a study of the structure of the oxygen absorption edge caused by the interstellar medium. More recently, it was also detected in the Cyg X-2 spectrum [24] in the observations that allowed the first spatially-resolved spectroscopic study of a scattering dust halo, as well as in the absorption towards LMC X-3 [25] and the recently discovered black hole candidate XTE J1817-330 [13].

To check the interstellar origin of the OI K $\alpha$ line observed in the RGS spectra of GRO J165540 , we looked for a correlation of the equivalent width of the line and the hydrogen column density, using the above-mentioned works (Fig. 3, left panel). We find that the equivalent width of the $\mathrm{OI} \mathrm{K} \alpha$ line, $\mathrm{EW}$, is indeed correlated with the column density, $\mathrm{N}_{\mathrm{H}}$, as $\mathrm{EW}(\mathrm{eV})=0.5( \pm 0.1)+$ $2.8( \pm 0.3) \times 10^{-22} \mathrm{~N}_{\mathrm{H}}\left(\mathrm{cm}^{-2}\right)$. The values observed for GRO J1655-40 fall well within this correlation for the ISM. We find that an alternative correlation with a zero regression constant could be $\mathrm{EW}(\mathrm{eV})=3.6( \pm 1.6) \times 10^{-22} \mathrm{~N}_{\mathrm{H}}\left(\mathrm{cm}^{-2}\right)$, but this would underestimate the equivalent width for the sources with hydrogen column density less than $5 \times 10^{21} \mathrm{~cm}^{-2}$. 


\subsection{A highly ionized wind}

Clear structured residuals also appear in the EPIC-pn spectrum at $\sim 6.8$ and $\sim 7.9 \mathrm{keV}$, corresponding to absorption features in the Fe $\mathrm{K}$ region (see Fig. 通, right panel). In all three March observations, the energy of the lines is the same, corresponding to the $\mathrm{K} \alpha$ and $\mathrm{K} \beta$ lines of either blended Fe XXV and Fe XXVI, or blueshifted Fe XXV (Table 2). Similar features had been previously detected in GRO J1655-40 with ASCA observations in 1994-1996 [26]. Assuming that the absorbing plasma is photoionized, the ionization state of the observed elements provides information on the conditions and location of the absorber. The presence of He-like $\mathrm{Fe}$ ions indicates an ionization parameter $\xi=\mathrm{L} / \mathrm{nr}^{2} \sim 10^{3} \mathrm{erg} \mathrm{cm} \mathrm{s}^{-1}[27$ ]. The lack of lower ionization absorption implies that the absorber cannot cover a large extent.

The equivalent width of the Fe lines increases between the first and the last March observations. This may be pointing out an increase either in the total column density or in the ionization state of the gas. From ASCA observations performed in 1994-1996, the iron column density of the plasma was determined to be $10^{19}-10^{20} \mathrm{~cm}^{-2}$ from the observed equivalent width and the curve of growth of the Fe XXV K $\alpha$ line [26], which relates the expected equivalent width to the iron column density. Using the curve of growth in [26] and assuming the detected features are only Fe XXV, our equivalent width of the Fe XXV K $\alpha$ line (between 50 and $160 \mathrm{eV}$ ) corresponds to a column density of $10^{19}$ and $5 \times 10^{20} \mathrm{~cm}^{-2}$, which is similar to the values found from ASCA observations [26]. Assuming cosmic abundances, this corresponds to a hydrogen column density in the range $2 \times 10^{23}-10^{25} \mathrm{~cm}^{-2}$. Since the observed absorption lines may be a blend of Fe XXV and Fe XXVI, these must be taken as upper limits. In addition, for a hydrogen column density higher than $10^{24} \mathrm{~cm}^{-2}$, the line absorber would be optically thick to Thomson scattering. We consider this to be unlikely and, taking into account the errors in the equivalent widths, the column density could then be in the range $2 \times 10^{23}-10^{24} \mathrm{~cm}^{-2}$ in all three observations.

With the photoionization parameter, $\xi \sim 10^{3} \mathrm{~cm} \mathrm{~s}^{-1}$, and the flux detected above $\sim 9 \mathrm{keV}$ (X-rays photoionizing He-like iron ions), $\mathrm{L}_{\geq 9 \mathrm{keV}} \sim 5 \times 10^{36} \mathrm{erg} \mathrm{s}^{-1}$ (for a distance of $3.2 \mathrm{kpc}$ ),
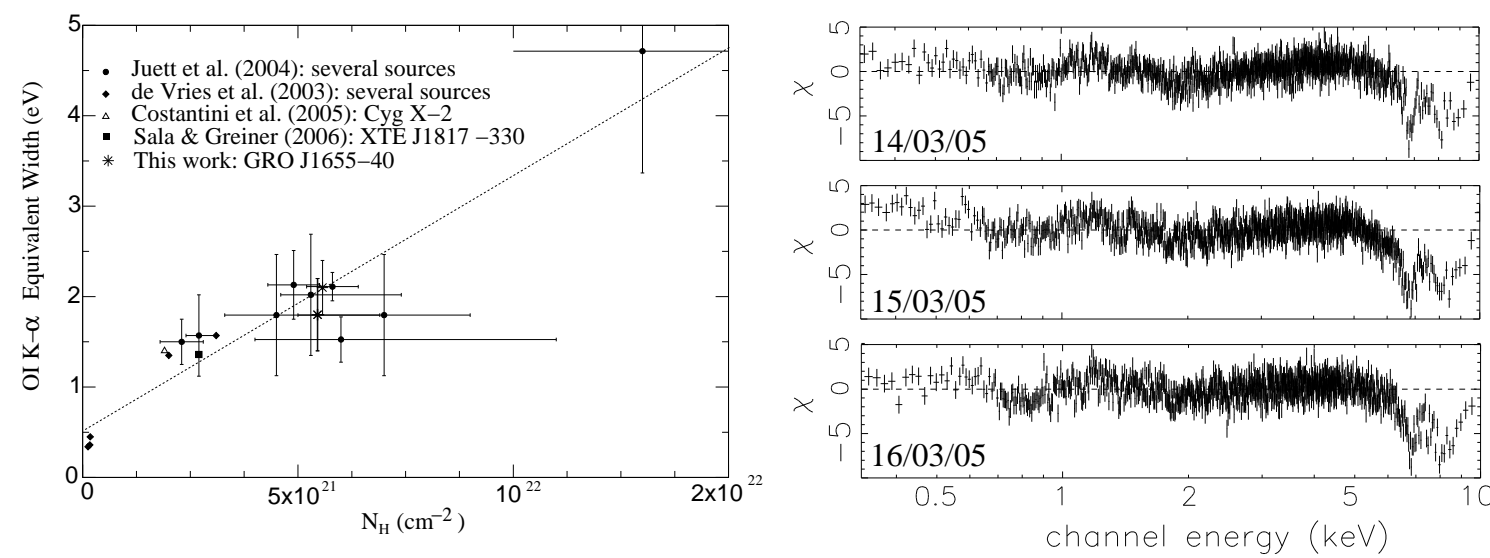

Figure 3: Left panel:OI K- $\alpha$ line equivalent width versus hydrogen column density for several galactic sources. The dashed line indicates an approximate linear correlation, $\mathrm{EW}(\mathrm{eV})=0.5+2.8 \times 10^{-22} \mathrm{~N}_{\mathrm{H}}\left(\mathrm{cm}^{-2}\right)$. Right panel:EPIC pn residuals of GRO J1655-40 spectra of our three March 2005 observations at zero velocity, after fitting an absorbed multi-temperature disk model. 
Table 2: Absorption features in EPIC-pn spectra

\begin{tabular}{|c|c|c|c|}
\hline Parameters & 14 March 2005 & 15 March 2005 & 16 March 2005 \\
\hline Line energy (keV) & $6.82 \pm 0.06$ & $6.71 \pm 0.06$ & $6.75 \pm 0.05$ \\
\hline $1 \sigma$ line width $(e V)$ & $110_{-50}^{+100}$ & $200 \pm 100$ & $250 \pm 50$ \\
\hline Equivalent width (eV) & $60_{-10}^{+30}$ & $100 \pm 60$ & $110 \pm 30$ \\
\hline Identification $^{\mathrm{a}}$ & \multicolumn{3}{|c|}{$\mathrm{Fe} X X V \mathrm{~K} \alpha\left(\mathrm{E}_{0} 6.700 \mathrm{keV}\right) / \mathrm{Fe} \mathrm{XXVI} \mathrm{K} \alpha\left(\mathrm{E}_{0} 6.952 / 6.973 \mathrm{keV}\right)$} \\
\hline $\mathrm{v}\left(\mathrm{km} \mathrm{s}^{-1}\right)^{\mathrm{b}}$ & $-5200 \pm 2600$ & $-400 \pm 2700$ & $-2200 \pm 2200$ \\
\hline Line energy $(\mathrm{keV})$ & $8.04 \pm 0.06$ & $7.85 \pm 0.15$ & $7.9 \pm 0.1$ \\
\hline $1 \sigma$ line width $(\mathrm{eV})$ & $100_{-50}^{+100}$ & $700 \pm 200$ & $600 \pm 200$ \\
\hline Equivalent width (eV) & $54_{-25}^{+1}$ & $230_{-50}^{+20}$ & $240 \pm 40$ \\
\hline Identification $^{\mathrm{a}}$ & \multicolumn{3}{|c|}{$\mathrm{Fe} X X V \mathrm{~K} \beta\left(\mathrm{E}_{0} 7.872 / 7.881 \mathrm{keV}\right) / \mathrm{Fe} \mathrm{XXVI} \mathrm{K} \beta\left(\mathrm{E}_{0} 8.246 / 8.252 \mathrm{keV}\right)$} \\
\hline $\mathrm{v}\left(\mathrm{km} \mathrm{s}^{-1}\right)^{\mathrm{b}}$ & $-5900 \pm 2200$ & $1200 \pm 5700$ & $-700 \pm 3800$ \\
\hline
\end{tabular}

a Based on CHIANTI data base [30, 31].

$\mathrm{b}$ Blueshift with respect to Fe XXV lines.

the column density indicates a distance to the central source between 50000 and $200000 \mathrm{~km}$. Assuming that the disk radius is $70 \%$ of the Roche lobe radius, i.e., $4 \times 10^{6} \mathrm{~km}$, the Fe XXV absorber extends over less than $5 \%$ of the disk size.

A Chandra observation of GRO J1655-40 was performed on 1 April 2005, two weeks after our last XMM-Newton observation [28]. The HETGS spectrum shows 90 absorption lines significant at the $5 \sigma$ level of confidence or higher. From all of these, only the Fe XXV $\mathrm{K} \alpha$ and $\mathrm{K} \beta$ lines are also present in our March observations. We computed the upper limit for the flux of some of the strongest lines detected by Chandra in the RGS energy range, in particular, for the Fe XXIV 5p, $4 p$, and $3 p$ lines at 7.159, 7.979, 10.604, and 10.649 A. Fixing the energy and FWHM of the lines to the values obtained with Chandra [28], we obtain the following upper limits at $3 \sigma$ confidence level for the flux of the lines: 4.2, 0.36, 1.4, and $1.3\left(\times 10^{-3} \mathrm{phcm}^{-2} \mathrm{~s}^{-1}\right)$, for the observation on 15 March 2005. These upper limits are between a factor 3 and 30 smaller than the fluxes measured in the Chandra spectrum and suggest a change in the ionization state between the two observation periods.

\subsection{Orbital dependence and inclination of the binary system}

Since the orbital period of GRO J1655-40 is $\sim 2.6$ days and our three March observations were taken in one day intervals, we cover approximately one whole orbital cycle. We can extrapolate the ephemeris obtained from the optical light-curve [8] to determine the orbital phase of our observations (error is 0.08 in all cases): $0.31-0.40$ (27 February), 0.17-0.24 (14 March), 0.55-0.62 (15 March), and 0.93-0.00 (16 March).

Given the inclination of the system (between $\sim 70^{\circ}$, [8, 29]; and $\sim 85^{\circ}$ [5]), an orbital phase close to zero corresponds to having the donor star situated closer to the observer, while the disk would be in front of the secondary star in phase 0.5 . This means that if any of the observed spectral features were arising from the illuminated face of the secondary star, it would have its maximum at 
phase 0.5 and would not be present at phase zero. This is not the case in our observations. Another possible orbital effect could be that the disk emission were absorbed by the stellar wind of the secondary star, which would produce increased absorptions close to phase zero, i.e. on $16 \mathrm{March}$, which is also not the case.

Finally, neither dips nor eclipses were observed in the disk thermal emission at any of the phases. Given the size of the donor star, $\sim 5 \mathrm{R}_{\odot}$ [8], the binary separation $\left(1.17 \times 10^{12} \mathrm{~cm}\right)$, and assuming that the soft X-ray emission originates in the central $200000 \mathrm{~km}$ of the disk (see Sect. 4.1, the duration of a possible eclipse of the soft X-ray emission by the donor star would last more than 2.5 hours (i.e., a change in the orbital phase of 0.04 ) close to phase zero. This should have been clearly visible during our 16 March observation.

The fact that no orbital modulation was observed in the X-ray spectra provides an upper limit for the inclination of the system. With the parameters mentioned above for the sizes of the system, the inclination must be smaller than $73^{\circ}$ (for the innermost $200000 \mathrm{~km}$ of the disk surface to be visible in all orbital phases). This limit is in agreement with the inclination determined from the optical light-curve in [8], $69.5 \pm 0.8$, and in [29], $67.2 \pm 3.5$.

\section{Summary}

GRO J1655-40 was observed with XMM-Newton during the low/hard state in February 2005 and in the soft/high state in March 2005. The continuum spectrum is dominated by an absorbed power law in the low/hard state and by a multicolor disk blackbody in the soft/high state, with the maximum temperature increasing during the high state. In addition, the X-ray spectrum during the March observations shows absorption lines of Fe XXV, possibly blended with Fe XXVI, indicating the presence of a highly ionized absorber with an ionization parameter of $1000 \mathrm{erg} \mathrm{cm} \mathrm{s}^{-1}$, extending less than $200000 \mathrm{~km}$ from the central source, and corresponding to $5 \%$ of the accretion disk.

Photoionized absorbers are seen in AGN as well, also with expansion velocities. The detection of photoionized winds in microquasars is then another similarity between these two kinds of systems. Nevertheless, winds in AGN are not uniform, but distributed instead in clouds of material. If this were the case in GRO J1655-40, the absorption systems would go in and out of the line of sight and cause temporal variations in the absorption features, which was not observed in our XMM-Newton observations.

\section{Acknowledgements}

The XMM-Newton project is an ESA Science Mission with instruments and contributions directly funded by ESA Member States and the USA (NASA), and partially supported by BMWI/DLR (FKZ 50 OX 0001), the Max-Planck Society, and the Heidenhain-Stiftung. We acknowledge the RXTE/ASM team for making quick-look results available for public use. This research has made use of public RXTE data obtained through the HEASARC Online Archive, provided by the NASA/GSFC.

\section{References}

[1] S.N. Zhang, C.A. Wilson, B.A. Harmon et al., X-Ray Nova in Scorpius, IAUC 6046 (1994) 
[2] F. Mirabel \& L.F.Rodriguez, A Superluminal Source in the Galaxy, Nature 371 (1994), 46

[3] S.N. Zhang, W. Cui, \& W. Chen, Black Hole Spin in X-Ray Binaries: Observational Consequences, ApJ 482 (1997), L155

[4] S.J. Tingay, D.L. Jauncey, R.A. Preston et al, Relativistic Motion in a Nearby Bright X-Ray Source, Nature 374 (1995), 141

[5] R.M. Hjellming \& M.P. Rupen, Episodic Ejection of Relativistic Jets by the X-Ray Transient GRO J1655-40, Nature 375 (1995), 464

[6] J. Greiner, P. Predehl, \& M. Pohl, ROSAT observations of GRO J1655-40, A\&A 297 (1995), L67

[7] C. Foellmi, E. Depagne, T.H. Dall \& F. Mirabel, On the distance of GRO J1655-40, A\&A 457 (2006), 249

[8] J.A. Orosz \& C.D. Bailyn, Optical Observations of GRO J1655-40 in Quiescence. I. A Precise Mass for the Black Hole Primary, ApJ 477 (1997), 876

[9] C.B. Markwardt \& J.H. Swank, New Outburst of GRO J1655-40?, ATel 414 (2005)

[10] J. Homan, J. Miller, R. Wijnands \& W.Lewin, GRO J1655-40 enters a highly-variable, high-luminosity state, ATel 487 (2005)

[11] C. Brocksopp, K.E. McGowan, H. Krimm et al, The 2005 outburst of GRO J1655-40: spectral evolution of the rise, as observed by Swift, MNRAS, 365 (2006), 1203

[12] J. Homan, GRO J1655-40 rapidly evolving, ATel 440 (2005)

[13] G. Sala, J. Greiner, J. Vink et al, The highly ionized disk wind of GRO J1655-40, A\&A, 461 (2007), 1049

[14] M.D. Caballero, E. Kuulkers, P. Kretschmar et al, TOO observations of GRO J1655-40 in outburst with INTEGRAL, in proceedings of the 6th INTEGRAL Workshop, eds. Sunyaev, Grebenev, Winkler; ESA/SP-622, in press; astro-ph/0609491.

[15] K. Makishima, Y. Maejima, K. Mitsuda, et al, Simultaneous X-ray and optical observations of $G X$ 339-4 in an X-ray high state, ApJ 308 (1986), 635

[16] D.A. Verner, G.J. Ferland, K.T. Korista \& D.G. Yakovlev, Atomic Data for Astrophysics. II. New Analytic FITS for Photoionization Cross Sections of Atoms and Ions, ApJ 465 (1996), 487

[17] J. Wilms, A. Allen, \& R. McCray, On the Absorption of X-Rays in the Interstellar Medium, ApJ 542 (2000), 914

[18] F. Paerels, A.C. Brinkmann, R.L.J. van der Meer et al., Interstellar X-Ray Absorption Spectroscopy of Oxygen, Neon, and Iron with the CHANDRA LETGS Spectrum of X0614+091, ApJ 546 (2001), 338

[19] N. Schulz, W. Cui, C.R. Canizares, et al., The First High-Resolution X-Ray Spectrum of Cygnus X-1: Soft X-Ray Ionization and Absorption, ApJ 565 (2002), 1141

[20] J. Wilms, A.M. Juett, N. Schulz, \& M.A. Nowak, 2007, in preparation (http://astro.uni-tuebingen.de/ wilms/research/tbabs/)

[21] A.M. Juett, N.S. Schulz, \& D. Chakrabarty, High-Resolution X-Ray Spectroscopy of the Interstellar Medium: Structure at the Oxygen Absorption Edge, ApJ 612 (2004), 308

[22] A.M. Juett, N.S. Schulz, D. Chakrabarty, \& T.W. Gorczyca, High-Resolution X-Ray Spectroscopy of the Interstellar Medium. II. Neon and Iron Absorption Edges, ApJ 648 (2006), 1066 
[23] C.P. de Vries, J.W. den Herder, J.S. Kaastra, et al., The interstellar oxygen-K absorption edge as observed by XMM-Newton. Separation of instrumental and interstellar components, A\&A 404 (2003), 959

[24] E. Costantini, M.J. Freyberg, \& P. Predehl, Absorption and scattering by interstellar dust: an XMM-Newton observation of Cyg X-2, A\&A 444 (2005), 187

[25] Q.D. Wang, Y. Yao, T.M. Tripp, et al., Warm-Hot Gas in and around the Milky Way: Detection and Implications of O VII Absorption toward LMC X-3, ApJ 635 (2005), 386

[26] Y. Ueda, H. Inoue, Y. Tanaka et al., Detection of Absorption-Line Features in the X-Ray Spectra of the Galactic Superluminal Source GRO J1655-40, ApJ 492 (1998), 782

[27] T. Kallman, D. Liedahl, A. Osterheld et al., Photoionization Equilibrium Modeling of Iron L Line Emission, ApJ 465 (1996), 994

[28] J. Miller, J. Raymond, A. Fabian et al., The magnetic nature of disk accretion onto black holes, Nature 441 (2006), 953

[29] E. van der Hooft, M. Heemskerk, F. Alberts, et al., The quiescence optical light curve of Nova Scorpii 1994 (=GRO J1655-40), A\&A, 329 (1998), 538

[30] K.P. Dere, E. Landi, H.E. Mason, B.C. Monsignori Fossi \& P.R. Young.,CHIANTI - an atomic database for emission lines, A\&AS 125 (1995), 149

[31] E. Landi, G. Del Zanna, P.R. Young, et al. CHIANTI-An Atomic Database for Emission Lines. VII. New Data for X-Rays and Other Improvements, ApJS 162 (2006), 261 